The influence of transformations on the h-index and the g-index Peer-reviewed author version

EGGHE, Leo (2008) The influence of transformations on the h-index and the g-index. In: JOURNAL OF THE AMERICAN SOCIETY FOR INFORMATION SCIENCE AND TECHNOLOGY, 59(8). p. 1304-1312.

DOI: $10.1002 /$ asi.20823

Handle: http://hdl.handle.net/1942/1792 


\title{
The influence of transformations on the h-index and the g-index
}

by

\author{
L. Egghe
}

Universiteit Hasselt (UHasselt), Campus Diepenbeek, Agoralaan, B-3590 Diepenbeek, Belgium $^{1}$

and

Universiteit Antwerpen (UA), Campus Drie Eiken, Universiteitsplein 1, B-2610 Wilrijk, Belgium

leo.egghe@uhasselt.be

\section{$\underline{\text { ABSTRACT }}$}

In a previous paper we introduced a general transformation on sources and one on items in an arbitrary information production process (IPP). In this paper we investigate the influence of these transformations on the h-index and on the g-index. General formulae that describe this influence are presented. These are applied to the case that the size-frequency function is Lotkaian (i.e. is a decreasing power function). We further show that the h-index of the transformed IPP belongs to the interval bounded by the 2 transformations of the h-index of the original IPP and we also show that this property is not true for the g-index.

\footnotetext{
1 Permanent address

Key words and phrases: h-index, g-index, transformation, Lotka, Zipf.
} 


\section{Introduction}

We suppose that we have a general information production process (IPP) with $\mathrm{f}:\left[\mathrm{a}, \rho_{\mathrm{m}}\right]{ }^{\circledR} \mathrm{i}^{+}, \mathrm{j}{ }^{\circledR} \mathrm{f}(\mathrm{j})$ as size-frequency function and with $\mathrm{F}:[0, \mathrm{~T}]{ }^{\circledR} i^{+}, \mathrm{r}{ }^{\circledR} \mathrm{F}(\mathrm{r})$ as rankfrequency function. Here $\mathrm{j}$ denote item densities and $\mathrm{r}$ rank densities; $\mathrm{a}$ and $\rho_{\mathrm{m}}$ are the minimum and maximum item densities and $\mathrm{T}$ denotes the total number of sources. We will limit ourselves to $\mathrm{a}=1$. Note that $\mathrm{F}$ usually is denoted by $\mathrm{g}$ (see Egghe (2005)) but to avoid confusion with the g-index we denote the rank-frequency function by $\mathrm{F}$.

Such a general IPP can be transformed in many ways, hereby describing a possible evolution of this IPP into another one, which we will denote by using asterisks * : size-frequency

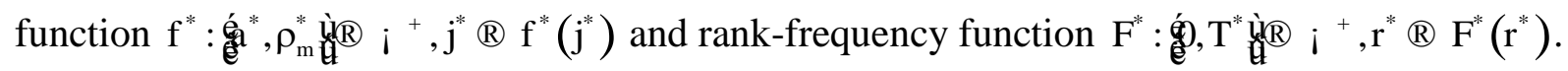

A very general way of describing the evolution from one IPP to another one is by applying two transformations: one on the sources:

$$
\begin{aligned}
& \psi:[0, \mathrm{~T}] \circledR \stackrel{g}{g}, \mathrm{~T}^{*} \text { ไ่̀ } \\
& r{ }^{\circledR} r^{*}=\psi(r)
\end{aligned}
$$

( $\psi$ differentiable and increasing $\psi(0)=0$ and $\psi(\mathrm{T})=\mathrm{T}^{*}$ ) and one on the items:

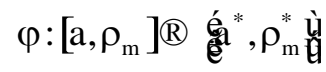

$$
\begin{aligned}
& \mathrm{j}^{\circledR} \mathrm{j}^{*}=\varphi(\mathrm{j})
\end{aligned}
$$

( $\varphi$ differentiable and increasing $\varphi(\mathrm{a})=\mathrm{a}^{*}, \varphi\left(\rho_{\mathrm{m}}\right)=\rho_{\mathrm{m}}^{*}$ ). These two functions $\varphi$ and $\psi$ the define the new rank-frequency function $\mathrm{g}^{*}$ :

$$
\mathrm{F}^{*}\left(\mathrm{r}^{*}\right)=\mathrm{F}^{*}(\psi(\mathrm{r}))=\varphi(\mathrm{F}(\mathrm{r}))
$$


for $\mathrm{r} \hat{I}[0, \mathrm{~T}]$.

General properties are studied in Egghe (2007a) where one also presents a formula for the transformed size-frequency function $\mathrm{f}^{*}$. We proved the following result:

$$
f^{*}\left(j^{*}\right)=f(j) \frac{\psi^{\prime}\left(F^{-1}(j)\right)}{\varphi^{\prime}(j)}
$$

for $\mathrm{j}^{*}=\varphi(\mathrm{j})$ (we assume $\varphi$ strictly increasing so that $\left.\varphi^{\prime}(\mathrm{j})^{1} \quad 0\right)$. In the case that $\psi(\mathrm{r})=\operatorname{Ar}^{\mathrm{b}}$ and $\varphi(j)=\mathrm{Bj}^{\mathrm{c}}(\mathrm{A}, \mathrm{B}>0, \mathrm{~b}, \mathrm{c}>0)$ and in case $\mathrm{f}$ is the function of Lotka:

$$
f(j)=\frac{C}{j^{\alpha}}
$$

with $\rho_{\mathrm{m}}=¥$ we prove in Egghe (2007a) that

$$
f^{*}\left(j^{*}\right)=\frac{D}{j^{* \delta}}
$$

where

$$
\mathrm{D}=\frac{\mathrm{CAbE}^{(\mathrm{b}-1)(\alpha-1)} \mathrm{B}^{\frac{(\alpha-1) b}{\mathrm{c}}}}{\mathrm{c}}
$$

and where

$$
\delta=\frac{c+(\alpha-1) b}{c}
$$

and where $\mathrm{E}$ is the parameter in the rank-frequency function

$$
F(r)=\frac{E}{r^{\beta}}
$$


which is equivalent with (5) as is well-known; here

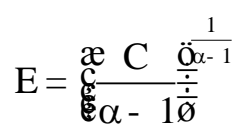

and

$$
\beta=\frac{1}{\alpha-1}
$$

- see Egghe (2005), Exercise II.2.2.6 or Egghe and Rousseau (2006a) where the complete proof is given.

The importance of these basic transformation results has been described in two papers. In Egghe and Rousseau (2006b) one shows that IPPs which grow such that the number of sources remains constant but where the number of items in each source grows extensively, have low Lotka exponents $\alpha$. This proof is given using formula (8) and confirmed in several examples of communities such as country sizes or municipality sizes or even database sizes. V. Cothey (2007) uses the same formula (8) to predict certain evolutions in (parts of) WWW.

We can now wonder what will be the effect of the above transformations on the h-index and g-index of an IPP. We first recall the definitions of these indexes. Hirsch (2005) defines the hindex as the largest rank $h$ of a source such that this source (and hence also the sources on a lower rank) has h or more items (Hirsch uses the paper-citation terminology hereby defining the h-index for an author). Since here we work in the continuous case where we only have continuous functions (hence where all values between two range values are attained) we have the following easy definition of the h-index:

$$
\mathrm{F}(\mathrm{h})=\mathrm{h}
$$

In Egghe and Rousseau (2006a) we show that h always uniquely exists. An alternative definition is 


$$
\grave{O}_{h}{ }^{*} f(j) d j=h
$$

as is readily seen.

The h-index, introduced only 2 years ago, has become a real hype in and even outside informetrics: Ball (2005, 2007), Bornmann and Daniel (2005, 2007a), Braun, Glänzel and Schubert $(2005,2006)$ (introducing the h-index for journals, yielding a new journal indicator to be preferred above the impact factor - see Miller (2006)), Glänzel (2006a,b), van Raan (2006), Bar-Ilan (2006), Rousseau (2007a), Burrell (2007a,b), Glänzel and Persson (2005), Egghe (2007b), Saad (2006), Oppenheim (2007), Hirsch (2007), Barendse (2007), Wan, Hua and Rousseau (2007), Rao and Rousseau (2007), Vinkler (2007), Vanclay (2007) and see also the papers in the special issue on the Hirsch index in Journal of Informetrics 1(3), 2007: Schubert and Glänzel (2007), Beirlant, Glänzel, Carbonez and Leemans (2007), Costas and Bordons (2007) and Bornmann and Daniel (2007b).

Banks (2006) introduces the interesting notion of the h-index for topics and compounds - see also Egghe and Rao (2007a) and the STIMULATE6 Group (2007). Schubert (2007) and Prathap (2006) introduce h-indices for institutions via the notion of "successive h-indices" (see also Egghe (2007c) and Egghe and Rao (2007b)). Let us, finally note that both the Web of Science and Scopus offer the h-index in their databases (remarkably quick after its introduction in 2005!).

According to Egghe (2006b) - see also Egghe (2006a,c), the h-index has (at least) one disadvantage: it does not take into account the exact number of citations of the first $h$ papers. In other words, once a paper belongs to the $h$ most cited papers, it does not matter anymore how much citations it actually received, even when this number can be very high. Therefore Egghe defined the g-index as the highest rank $g$ of a source such that all the sources on this and lower ranks have together, at least $\mathrm{g}^{2}$ items. In the continuous model this gives the following defining equation for the g-index:

$$
\mathrm{G}(\mathrm{g})=\mathrm{O}_{0}^{\mathrm{g}} \mathrm{F}(\mathrm{r}) \mathrm{dr}=\mathrm{g}^{2}
$$


Alternatively, $g$ is defined via (the less simple) equations

$$
\begin{aligned}
& \grave{O}_{j}^{¥} f\left(j^{\prime}\right) d j^{\prime}=g \\
& \grave{O}_{j}^{¥} j^{\prime} f\left(j^{\prime}\right) d j^{\prime}=g^{2}
\end{aligned}
$$

In Egghe (2006b) it is shown that also g uniquely exists.

Of course we assume here that $I £ T^{2}$ ( $I=$ total number of items, $T=$ total number of sources). This is not always true since, if $\rho_{\mathrm{m}}=¥$ (what we suppose in this paper), and if $\mathrm{f}$ is Lotkaian (equation (5)) with $\alpha>2$, then it follows from

$$
I=\grave{O}_{1}^{¥} j f(j) d j
$$

and

$$
T=\grave{O}_{1} f(j) d j
$$

that (see also Proposition II.2.1.1.1 in Egghe (2005))

$$
\mathrm{I}=\frac{\alpha-1}{\alpha-2} \mathrm{~T}
$$

(I is called A in Egghe (2005)), so I can be larger than $T^{2}$ if $\alpha>2$ is close enough to 2. In practise one can add fictitious sources with 0 items so that $\mathrm{g}$ can be defined beyond $\mathrm{T}$. But in this theoretical paper this is no problem: we just assume that $\mathrm{I}<\mathrm{T}^{2}$ which implies

$$
\frac{\alpha-1}{\alpha-2}<\mathrm{T}
$$

hence 


$$
\alpha>\frac{2 \mathrm{~T}-1}{\mathrm{~T}-1}
$$

which is almost always true if $\alpha>2$ since T usually is large.

It is further trivial that $\mathrm{g}^{3} \mathrm{~h}$ in each IPP.

In Egghe $(2006 b, c)$ it is shown that the g-index has more discriminatory power than the hindex. This finding was also confirmed in Schreiber (2007) and Tol (2007). The h- and gindex (and some other indices) can be calculated using the software program "Publish or Perish" (see http://www.harzing.com/pop.htm).

The above explanations of the importance of transformations in IPPs and of the importance of the h- and the g-index should make it clear that the study of the influence of transformations on the h- and g-index is equally important.

In the next section the analogue definitions of the $\mathrm{h}$ - and g-index in the transformed system will be studied and calculated in general systems and in case we have a Lotkaian system (5).

In the third section we prove, denoting by $\mathrm{h}$ the $\mathrm{h}$-index in the original system and by $\mathrm{h}^{*}$ the h-index in the transformed system, that always

$$
\varphi(h) £ h^{*} £ \psi(h)
$$

or

$$
\psi(h) £ h^{*} £ \varphi(h)
$$

(with strict inequalities if $\psi(\mathrm{h})^{1} \varphi(\mathrm{h})$ ). We also show by example that none of the above double inequalities are true for the g-index.

Finally we present conclusions and suggestions for further research. 


\section{General equations for the $h$ - and g-index in general transformed IPPs and in Lotkaian systems}

In the sequel we will denote by $\mathrm{h}$ and $\mathrm{g}$ the $\mathrm{h}$-index and $\mathrm{g}$-index in the original IPP and by $\mathrm{h}^{*}$ and $\mathrm{g}^{*}$ the h-index and g-index in the transformed IPP, the transformations being given by (1), (2) and (3).

\section{II.1 General equations for $\mathrm{h}^{*}$}

Theorem II.1: The transformation formula for $\mathrm{h}$ is

$$
\grave{O}_{j^{\prime}=\varphi^{-1}\left(h^{*}\right)}^{¥} f\left(j^{\prime}\right) \psi^{\prime}\left(F^{-1}\left(j^{\prime}\right)\right) d j^{\prime}=h^{*}
$$

For the proof: see Appendix A

Let us now illustrate how these basic equations can be used in the concrete calculation of $\mathrm{h}^{*}$.

\section{II.2 Calculation of $\mathrm{h}^{*}$ given that the original IPP is Lotkaian}

Theorem II.2: We suppose that $f$ is as in (5), the law of Lotka. We will again suppose that the transformations are increasing power laws, an important case: for $\mathrm{A}, \mathrm{B}>0, \mathrm{~b}, \mathrm{c}>0$ :

$$
\begin{gathered}
\mathrm{j}^{*}=\varphi(j)=B j^{c} \\
r^{*}=\psi(r)=A r^{b}
\end{gathered}
$$

Then we have that

$$
\mathrm{h}^{*}=\mathrm{B}^{\frac{\delta-1}{\delta}} \mathrm{T}^{* \frac{1}{\delta}}
$$

with $\delta$ as in (8).

For the proof we refer to Appendix B. 
This result generalizes the result

$$
\mathrm{h}=\mathrm{T}^{\frac{1}{\alpha}}
$$

obtained in Egghe and Rousseau (2006a).

We now turn our attention to the calculation of the g-index.

\section{II.3 General equation for $\mathrm{g}^{*}$}

For the sake of simplicity we will only use equation (14). For the transformed system this gives

$$
\mathrm{O}_{0}^{g^{*}} \mathrm{~F}^{*}\left(\mathrm{r}^{*}\right) \mathrm{dr}^{*}=\mathrm{g}^{* 2}
$$

By (1) and (3) we have

$$
\grave{\mathrm{O}}_{0}^{\psi^{-1}\left(\mathrm{~g}^{*}\right)} \varphi(\mathrm{F}(\mathrm{r})) \psi^{\prime}(\mathrm{r}) \mathrm{dr}=\mathrm{g}^{* 2}
$$

which is the basic general equation for $\mathrm{g}^{*}$.

Again we will illustrate its use, given (5), (20) and (21).

\section{II.4 Calculation of $\mathrm{g}^{*}$ given that the original IPP is Lotkaian}

Theorem II.3: We suppose that $\mathrm{f}$ is as in (5) and $\varphi$ and $\psi$ are as in (20) and (21). Then

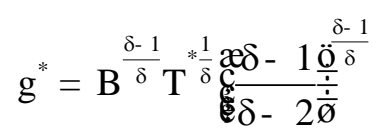

with $\delta$ as in (8).

For the proof we refer to Appendix C. 
Note that the requirement $\beta c+1-b<1$ is needed for the convergence of the integral. For the same reason one must require, in the original IPP, that $\alpha>2$ in order to be able to calculate the g-index g. If we work in systems with bounded densities then formula (9) is replaced by the function of Mandelbrot (see Egghe (2005)) and the restrictions can be dropped. We do not follow this approach since calculations become very intricate. Note also, as is readily seen, that restriction $\beta c+1-b<1$ is equivalent with $\delta>2$ (so exactly the same requirement as $\alpha>2$ in the original system).

Formula (26) could also have been obtained from (22) together with the result on the g-index, proved in Egghe (2006b) (in our notation):

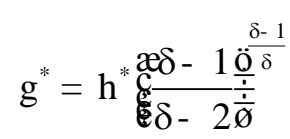

\section{Qualitative study of $\mathrm{h}^{*}$ and $\mathrm{g}^{*}$ in comparison with $h$ and $g$}

Since $\mathrm{h}^{*}$ and $\mathrm{g}^{*}$ are the $\mathrm{h}$-index and g-index of the transformed system it would be logical that one can prove relations with $\varphi(\mathrm{h})$ and $\psi(\mathrm{h})$, respectively $\varphi(\mathrm{g})$ and $\psi(\mathrm{g})$, the transformed values of $\mathrm{h}$ and $\mathrm{g}$, respectively the h-index and g-index of the original system. Note that $\mathrm{h}$ and $\mathrm{g}$, by definition, can be considered as arguments of $\varphi \underline{\text { and }} \psi$. This is clear from (12) for $\mathrm{h}$ and from (15) for $\mathrm{g}$ (implying $\mathrm{g} £ \mathrm{~T}$, assuming $\mathrm{I} £ \mathrm{~T}^{2}$ as we do in this paper) and since the argument of $\varphi$ is unbounded (since we assume $\rho_{\mathrm{m}}=¥$ in this paper).

We will now show that $\mathrm{h}^{*}$ is limited by $\varphi(\mathrm{h})$ and $\psi(\mathrm{h})$ but that this is not the case for $\mathrm{g}^{*}$ in relation with $\varphi(\mathrm{g})$ and $\psi(\mathrm{g})$.

We first prove a Lemma. 


\section{Lemma III.1:}

For all IPPs we have

$$
\mathrm{F}^{*}(\psi(\mathrm{h}))=\varphi(\mathrm{F}(\mathrm{h}))=\varphi(\mathrm{h})
$$

\section{Proof:}

This follows readily from (3) and by the fact that the h-index satisfies $F(h)=h$, by (12).

\section{Theorem III.2:}

For all IPPs we have

$$
h^{*}=\varphi(h)=\psi(h)
$$

or

$$
\varphi(h)<h^{*}<\psi(h)
$$

or

$$
\psi(h)<h^{*}<\varphi(h)
$$

For the proof we refer to Appendix D.

\section{Corollary III.3:}

For all IPPs we have

$$
\mathrm{h}=\varphi^{-1}\left(\mathrm{~h}^{*}\right)=\psi^{-1}\left(\mathrm{~h}^{*}\right)
$$

or

$$
\psi^{-1}\left(h^{*}\right)<h<\varphi^{-1}\left(h^{*}\right)
$$




$$
\varphi^{-1}\left(\mathrm{~h}^{*}\right)<\mathrm{h}<\psi^{-1}\left(\mathrm{~h}^{*}\right)
$$

\section{Proof:}

(31) follows from (28), (32) from (29) and (33) from (30) using that $\varphi$ and $\psi$ are increasing functions.

We will now show by example that Theorem III.2 (hence also Corollary III.3) is not true for the g-index.

\section{Example III.4}

Take $\psi=I d$, the identity function (hence $A=b=1$ and $T=T^{*}$ ) and let $\varphi(j)=j^{2}$ (hence $\mathrm{B}=1, \mathrm{c}=2$ ). Let the original IPP be Lotkaian with $\alpha=3.1$ (and $\rho_{\mathrm{m}}=¥$ ). We have

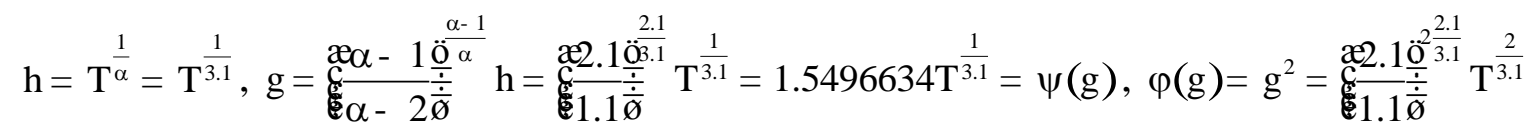

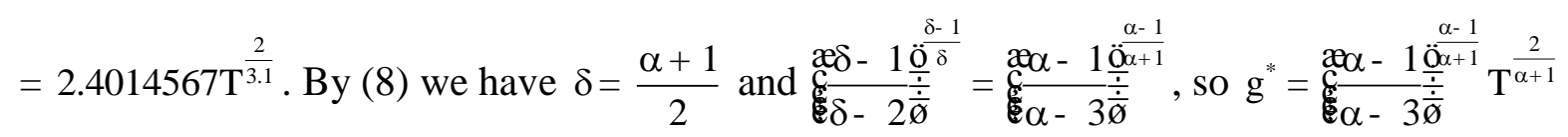

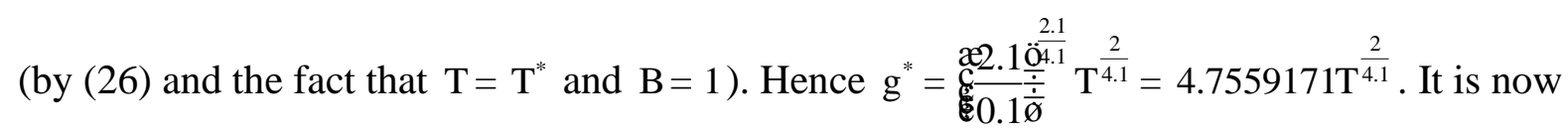
clear that $\psi(\mathrm{g})<\varphi(\mathrm{g})$ and $\mathrm{g}^{*}>\varphi(\mathrm{g})$ if $4.7559171 \mathrm{~T}^{\frac{2}{4.1}}>2.4014567 \mathrm{~T}^{\frac{2}{3.1}}$ which is true for $\mathrm{T}<76.896529$. So in all these cases

$$
\mathrm{g}^{*} \ddot{\mathrm{I}} \text { ù̀ } \psi(\mathrm{g}), \varphi(\mathrm{g}) \text { é }
$$

proving that Theorem III.2 is not true for the g-index. By interchanging $\varphi$ and $\psi$ we also see that the other inequalities in Theorem III.2 are also not true for the g-index. Note that in the above example

$$
\mathrm{h}^{*} \hat{\mathrm{I}} \mathrm{u} u(\mathrm{~h}), \varphi(\mathrm{h}) e_{\mathrm{e}}
$$


since

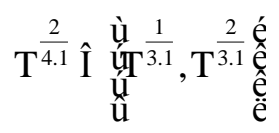

; in fact, for all $\alpha$

$$
\mathrm{T}^{\frac{2}{\alpha+1}} \hat{\mathrm{I}} \underset{\substack{\mathrm{u}_{\mathrm{u}} \\ \text { ù }}}{\frac{1}{\alpha}}, \mathrm{T}^{\frac{2}{\alpha}} \underset{\mathrm{e}}{\mathrm{e}}
$$

since $\alpha>1$, illustrating Theorem III.2. So we see that the factor

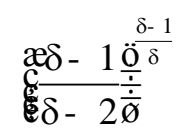

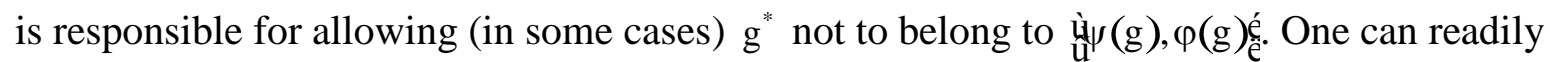
verify that, if $\alpha=10$ in the above example we now have that $\mathrm{g}^{*} \hat{\mathrm{I}} \underset{\mathrm{u}}{\mathrm{u}} \varphi(\mathrm{g}), \varphi(\mathrm{g}) \mathrm{e}_{\mathrm{e}}^{\text {showing that }}$ this case can happen too. Indeed, for $\psi$ and $\varphi$ as above we have

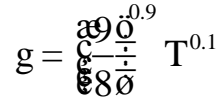

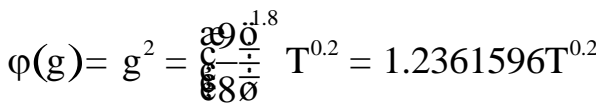

$$
\begin{aligned}
& \psi(g)=g=1.1118271 \mathrm{~T}^{0.1}
\end{aligned}
$$

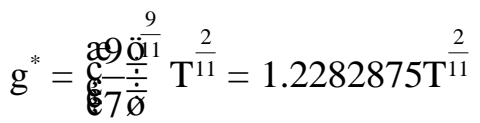

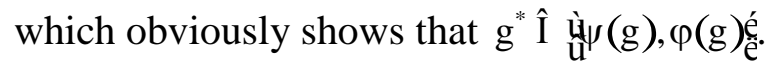


We can also give an example where

$$
\mathrm{g}^{* 1} \varphi(\mathrm{g})=\psi(\mathrm{g})
$$

showing that also (28) in Theorem III.2 is false for the g-index. Indeed, take $\varphi(j)=j^{2}$, $\psi(r)=r^{2}$ for all $\mathrm{j}, \mathrm{r}$. We know that in this case $\delta=\alpha($ since $\mathrm{b}=\mathrm{c}=2$ and see (8)). Hence

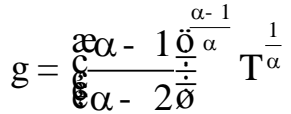

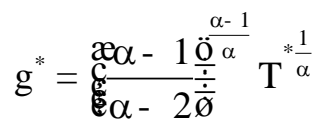

But $T^{*}=\psi(T)=T^{2}$, so

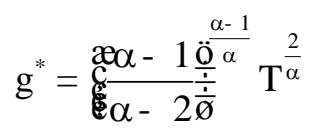

But

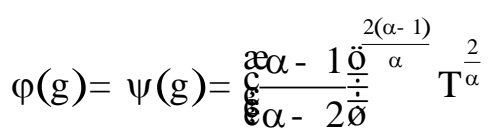

${ }^{1} \mathrm{~g}^{*}$. 


\section{Conclusions and suggestions for further}

\section{research}

We considered a very general double transformation on an IPP: $j{ }^{\circledR} \varphi(j)$ for the items and $r{ }^{\circledR} \psi(r)$ for the sources such that the rank-frequency function $\mathrm{F}^{*}$ of the transformed IPP relates to the rank-frequency function F of the original IPP as follows

$$
\mathrm{F}^{*}\left(\mathrm{r}^{*}\right)=\mathrm{F}^{*}(\psi(\mathrm{r}))=\varphi(\mathrm{F}(\mathrm{r}))
$$

for $\mathrm{r} \hat{\mathrm{I}}[0, \mathrm{~T}]$.

Based on this we show that the basic equation for the h-index $h^{*}$ of the transformed IPP is

$$
\grave{\mathrm{O}}_{\mathrm{j}^{\prime}=\varphi^{-1}\left(\mathrm{~h}^{*}\right)}^{¥} \mathrm{f}\left(\mathrm{j}^{\prime}\right) \psi^{\prime}\left(\mathrm{F}^{-1}\left(\mathrm{j}^{\prime}\right)\right) \mathrm{dj} \mathrm{j}^{\prime}=\mathrm{h}^{*}
$$

or, equivalently,

$$
\varphi^{-1}\left(\mathrm{~h}^{*}\right)=\mathrm{F}\left(\psi^{-1}\left(\mathrm{~h}^{*}\right)\right)
$$

For the g-index $\mathrm{g}^{*}$ of the transformed system we have the basic equation

$$
\grave{\mathrm{O}}_{0}^{\psi^{-1}\left(\mathrm{~g}^{*}\right)} \varphi(\mathrm{F}(\mathrm{r})) \psi^{\prime}(\mathrm{r}) \mathrm{dr}=\mathrm{g}^{* 2}
$$

These equations are then used to prove that

$$
\mathrm{h}^{*}=\mathrm{B}^{\frac{\delta-1}{\delta}} \mathrm{T}^{* \frac{1}{\delta}}
$$

if we have a Lotkaian system 


$$
f(j)=\frac{C}{j^{\alpha}}
$$

and where $\psi(r)=A r^{b}$ and $\varphi(j)=B j^{\mathrm{c}}$ and where

$$
\delta=\frac{c+(\alpha-1) b}{c}
$$

For $\mathrm{g}^{*}$ we proved

$$
\mathrm{g}^{*}=\frac{\mathfrak{c} \delta-1 \frac{\dot{\delta}}{\delta} \delta}{\frac{\delta-1}{\dot{\bar{\phi}}}} \mathrm{B}^{\frac{\delta-1}{\delta}} \mathrm{T}^{\frac{1}{\delta}}
$$

for $\delta>2$.

Finally we prove that

$$
h^{*}=\varphi(h)=\psi(h)
$$

or

$$
\varphi(h)<h^{*}<\psi(h)
$$

or

$$
\psi(h)<h^{*}<\varphi(h)
$$

for any IPP and we show that none of these inequalities or equalities are generally true for the g-index.

The transformations $\varphi$ and $\psi$ are generalizations of positive reinforcement of IPPs which belongs to linear three-dimensional informetrics theory (cf. Egghe (2005), Chapter 3 and Egghe (2004), Rousseau (1992)). We leave open to study linear three-dimensional 
informetrics theory from the point of view of the h- and g-index. More in particular it would be interesting to see if conclusions around the h-index and the g-index of the composed IPP can be drawn based on the h- and g-indexes of the composing IPPs.

Possibly also other transformations of IPPs can be studied and it would then be interesting to study the transformed h- and g-indexes based on the h- and g-indexes of the original IPP, as we studied here.

One referee asked the question if these results are true in the discrete setting (after all, $\mathrm{h}$ - and g-indices are calculated from discrete tables). Certainly the transformation formulae cannot be proved in the discrete setting. The reason why we work in the continuous setting is the calculability of the formulae. To give a simple example: formula (23):

$$
\mathrm{h}=\mathrm{T}^{\frac{1}{\alpha}}
$$

was proved first in Glänzel (2006b) in an approximative way but the continuous derivation, given in Egghe and Rousseau (2006a), is exact and more elegant.

It remains an interesting problem to prove (or disprove) the validity of one of the formulae (28), (29) or (30) in the discrete setting. To be honest, I do not know if the same result is valid in this discrete case and I have to leave it as an open problem.

\section{Appendix A: Proof of Theorem II.1}

\section{Using formula (13)}

Clearly, by (13), the analogously defining equation for $\mathrm{h}^{*}$ is

$$
\grave{\mathrm{O}}_{\mathrm{h}^{*}}^{*} \mathrm{f}^{*}\left(\mathrm{j}^{* \prime}\right) \mathrm{dj} \mathrm{j}^{*^{\prime}}=\mathrm{h}^{*}
$$

Using (4) yields

$$
\grave{\mathrm{O}}_{\mathrm{j}^{*}=\mathrm{h}^{*}} \underset{*}{*} \mathrm{f}\left(\mathrm{j}^{\prime}\right) \frac{\psi^{\prime}\left(\mathrm{F}^{-1}\left(\mathrm{j}^{\prime}\right)\right)}{\varphi^{\prime}\left(\mathrm{j}^{\prime}\right)} \mathrm{dj} \mathrm{j}^{*^{\prime}}=\mathrm{h}^{*}
$$


with $\mathrm{j}^{* \prime}=\varphi\left(\mathrm{j}^{\prime}\right)$, hence

$$
\begin{aligned}
& \grave{O}_{\mathrm{j}^{\prime}=\varphi^{-1}\left(\mathrm{~h}^{*}\right)}^{¥} \mathrm{f}\left(\mathrm{j}^{\prime}\right) \psi^{\prime}\left(\mathrm{F}^{-1}\left(\mathrm{j}^{\prime}\right)\right) \mathrm{dj^{ \prime }}=\mathrm{h}^{*} \\
& \grave{O}_{j^{\prime}=\varphi^{-1}\left(h^{*}\right)}^{¥} f\left(j^{\prime}\right) \psi^{\prime}\left(F^{-1}\left(j^{\prime}\right)\right) F^{\prime}\left(F^{-1}\left(j^{\prime}\right)\right) d \dot{G}^{-1}\left(j^{\prime}\right) \stackrel{\grave{k}=}{\dot{k}} h^{*} \\
& \grave{\mathrm{O}}_{\mathrm{k}^{\prime}=\mathrm{F}^{1}\left(\varphi^{-1}\left(\mathrm{~h}^{*}\right)\right)}^{¥} \mathrm{f}\left(\mathrm{F}\left(\mathrm{k}^{\prime}\right)\right) \psi^{\prime}\left(\mathrm{k}^{\prime}\right) \mathrm{F}^{\prime}\left(\mathrm{k}^{\prime}\right) \mathrm{dk^{ \prime }}=\mathrm{h}^{*}
\end{aligned}
$$

where $k^{\prime}=F^{-1}\left(j^{\prime}\right)$, hence $j^{\prime}=F\left(k^{\prime}\right)$. Finally:

$$
\begin{gathered}
\grave{\mathrm{O}}_{\mathrm{k}^{\prime}=F^{\prime}\left(\varphi^{-1}\left(\mathrm{~h}^{*}\right)\right)}^{¥} \mathrm{f}\left(\mathrm{F}\left(\mathrm{k}^{\prime}\right)\right) \psi^{\prime}\left(\mathrm{k}^{\prime}\right) \mathrm{d} \dot{\mathrm{F}}\left(\mathrm{k}^{\prime}\right) \stackrel{\mathrm{u}}{\mathrm{u}} \mathrm{h}^{*} \\
\grave{\mathrm{O}}_{\mathrm{j}^{\prime}=\varphi^{-1}\left(\mathrm{~h}^{*}\right)} \mathrm{f}\left(\mathrm{j}^{\prime}\right) \psi^{\prime}\left(\mathrm{F}^{-1}\left(\mathrm{j}^{\prime}\right)\right) \mathrm{j}^{\prime}=\mathrm{h}^{*}
\end{gathered}
$$

This is the defining equation for $\mathrm{h}^{*}$ in terms of the size-frequency function $\mathrm{f}$, the rankfrequency function $\mathrm{F}$ and the transformations $\varphi$ and $\psi$.

\section{Using equation (12)}

For the transformed IPP we have the analogue of (12): $\mathrm{h}^{*}$ is defined as

$$
\mathrm{F}^{*}\left(\mathrm{~h}^{*}\right)=\mathrm{h}^{*}
$$

which is, in terms of the functions of the original IPP and of the transformations, by (3):

$$
\mathrm{h}^{*}=\varphi\left(\mathrm{F}\left(\psi^{-1}\left(\mathrm{~h}^{*}\right)\right)\right)
$$


(note that, by (1), one is tempted to write $\psi(h)=h^{*}$ but this is not true if $h$ is the $h$-index of the original IPP - see further). Alternatively one can also use

$$
\varphi^{-1}\left(\mathrm{~h}^{*}\right)=\mathrm{F}\left(\psi^{-1}\left(\mathrm{~h}^{*}\right)\right)
$$

\section{Appendix B: Proof of theorem II.2}

\section{$\underline{\text { 1. Using formula (19) }}$}

Note that

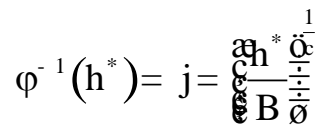

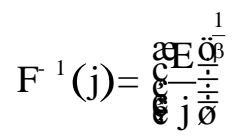

and

$$
\psi^{\prime}(\mathrm{r})=\mathrm{Abr}^{\mathrm{b}-1}
$$

This gives in (19), using also (5):

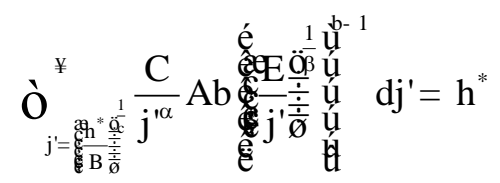

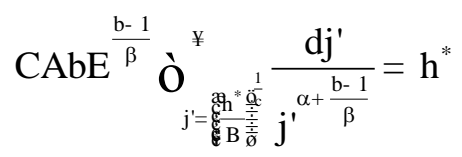

Hence $\left(\right.$ since $\alpha+\frac{b-1}{\beta}>1$ since $\alpha>1$ and $b>1$ ) 


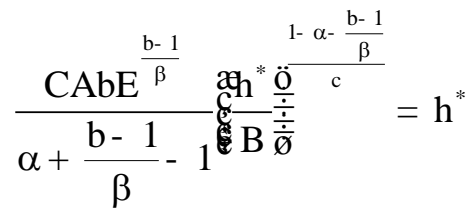

\section{Hence}

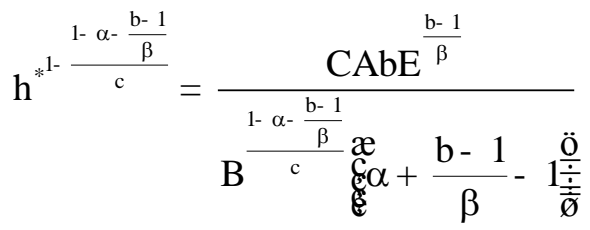

Note that

$$
1-\frac{1-\alpha-\frac{b-1}{\beta}}{c}=\frac{c+b(\alpha-1)}{c}=\delta
$$

by (11) and by notation (8). Further

$$
\begin{aligned}
& \frac{\mathrm{CAbE}^{\frac{\mathrm{b}-1}{\beta}}}{1-\alpha-\frac{\mathrm{b}-1}{\beta} \mathrm{x}} \mathrm{b}-1 \stackrel{\ddot{O}}{\mathrm{o}}=\frac{\mathrm{CAbE}^{(\mathrm{b}-1)(\alpha-1)} \mathrm{B}^{\delta-1}}{\mathrm{c}(\delta-1)}
\end{aligned}
$$

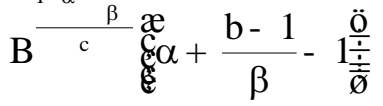

Hence we refound parameter D (see (7)): (B1) yields

$$
\mathrm{h}^{* \delta}=\frac{\mathrm{D}}{\delta-1}
$$

But

$$
\mathrm{T}^{*}=\mathrm{O}_{\mathrm{B}} \frac{\mathrm{D}}{\mathrm{j}^{* \delta}} \mathrm{dj} \mathrm{j}^{*}=\frac{\mathrm{D}}{\delta-1} \mathrm{~B}^{1-\delta}
$$

(since $j^{*}=\varphi(j)=B j^{c^{3}} B$ since $\left.j^{3} 1\right)$. So 


$$
\mathrm{h}^{* \delta}=\frac{\mathrm{T}^{*}}{\mathrm{~B}^{1-\delta}}
$$

Hence

$$
\mathrm{h}^{*}=\mathrm{B}^{\frac{\delta-1}{\delta}} \mathrm{T}^{* \frac{1}{\delta}}
$$

An equivalent calculation would be: follow the calculation of (6), (7), (8) in Egghe (2006a) and apply (23) with $\mathrm{T}^{*}$ and using (B2).

We will now show how we can use (A1) (or (A2)).

\section{Using formula (A2)}

Note that

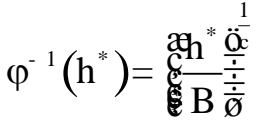

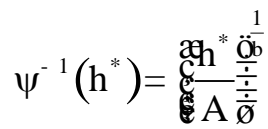

and that $\mathrm{F}$ satisfies (9) with $\beta$ as in (11). Hence (A2) gives

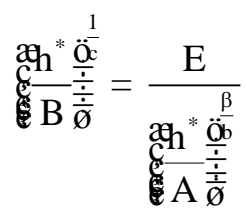

Use (10) to get

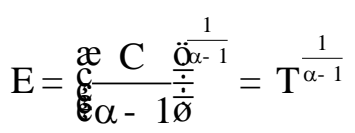


since

$$
T=\grave{O}_{1}^{*} f(j) d j=\grave{O}_{1} \frac{C}{j^{\alpha}} d j=\frac{C}{\alpha-1}
$$

since $\alpha>1$, T being the total number of sources. But

$$
\mathrm{T}^{*}=\psi(\mathrm{T})=\mathrm{AT}^{\mathrm{b}}
$$

since $\psi$ increases. (B5) and (B4) in (B3) yields

$$
\mathrm{h}^{*}=\mathrm{B}^{\frac{\frac{1}{\mathrm{c}}}{\frac{1}{\mathrm{c}^{2}+\frac{\beta}{\mathrm{b}}}}} \mathrm{T}^{\frac{\frac{1}{\mathrm{~b}(\alpha-1)}}{\mathrm{c}^{\frac{1}{\mathrm{~b}}+\frac{\beta}{\mathrm{b}}}}}
$$

which is readily seen to be the same as (22), using (11).

\section{Appendix C: Proof of Theorem II.3}

We have that

$$
\begin{aligned}
& \psi^{\prime}(\mathrm{r})=\mathrm{Abr}^{\mathrm{b}-1}
\end{aligned}
$$

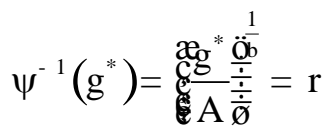

Hence we have the equation, based on (25)

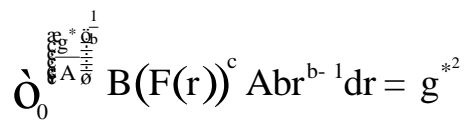

Using (9) gives

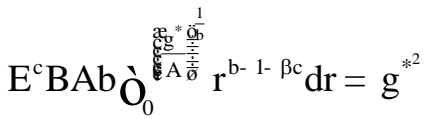


For $\beta c+1-b<1$ we have

$$
\frac{E^{c} B b A^{\frac{\beta c}{b}}}{b-\beta c} g^{* \frac{b-\beta c}{b}}=g^{*^{2}}
$$

Hence

$$
g^{{ }^{*}+\frac{\beta c}{b}}=\frac{B b A^{\frac{\beta c}{b}} E^{c}}{b-\beta c}
$$

Now we use (11) and (B5) yielding

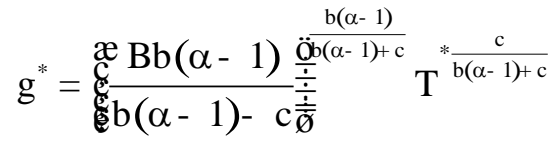

hence, using notation (8) for $\delta$ we find

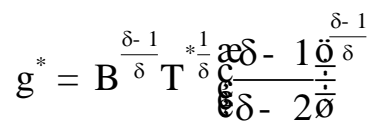

\section{Appendix D: proof of Theorem III.2}

(i) Let $h^{*}=\psi(h)$. Then, by definition of the h-index in both systems and by Lemma III.1 we have

$$
\begin{aligned}
h^{*}=F^{*}\left(h^{*}\right) & =F^{*}(\psi(h)) \\
& =\varphi(F(h))=\varphi(h)
\end{aligned}
$$

proving (28). 


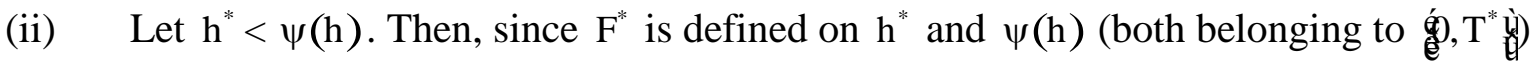
and since $\mathrm{F}^{*}$ strictly decreases, we have

$$
\mathrm{h}^{*}=\mathrm{F}^{*}\left(\mathrm{~h}^{*}\right)>\mathrm{F}^{*}(\psi(\mathrm{h}))
$$

Again invoking the above lemma yields

$$
\mathrm{h}^{*}>\varphi(\mathrm{h})
$$

hence proving (29).

(iii) Let $\mathrm{h}^{*}>\psi(\mathrm{h})$. By the same argument we have

$$
h^{*}=F^{*}\left(h^{*}\right)<F^{*}(\psi(h))
$$

Hence by the above Lemma:

$$
\mathrm{h}^{*}<\varphi(\mathrm{h})
$$

proving (30).

\section{$\underline{\text { References }}$}

P. Ball (2005). Index aims for fair ranking of scientists. Nature 436, 900, 2005.

P. Ball (2007). Achievement index climbs the ranks. Nature 448, 16 August 2007, 737, 2007.

M.G. Banks (2006). An extension of the Hirsch index: indexing scientific topics and compounds. Scientometrics 69(1), 161-168, 2006.

J. Bar-Ilan (2006). H-index for Price medallists revisited. ISSI Newsletter 2(1), 3-5, 2006. 
W. Barendse (2007). The strike rate index: a new index for journal quality based on journal size and the h-index of citations. Biomedical Digital Libraries 4(3), 2007. http://www.bio-diglib.com/content/4/1/3

J. Beirlant, W. Glänzel, A. Carbonez and H. Leemans (2007). Scoring research output using statistical quantile plotting. Journal of Informetrics 1(3), 185-192, 2007.

L. Bornmann and H.-D. Daniel (2005). Does the h-index for ranking of scientists really work? Scientometrics 65(3), 391-392, 2005.

L. Bornmann and H.-D. Daniel (2007a). What do we know about the h-index ? Journal of the American Society for Information Science and Technology 58(9), 1381-1385, 2007.

L. Bornmann and H.-D. Daniel (2007b). Convergent validation of peer review decisions using the h-index. Extent of and reasons for type I and type II errors. Journal of Informetrics 1(3), 204-213, 2007.

T. Braun, W. Glänzel and A. Schubert (2005). A Hirsch-type index for journals. The Scientist, 19(22), 8, 2005.

T. Braun, W. Glänzel and A. Schubert (2006). A Hirsch-type index for journals. Scientometrics 69(1), 169-173, 2006.

Q.L. Burrell (2007a). Hirsch's h-index: a stochastic model. Journal of Informetrics 1(1), 16$25,2007$.

Q.L. Burrell (2007b). On the h-index, the size of the Hirsch core and Jin's A-index. Journal of Informetrics 1(2), 170-177, 2007.

R. Costas and M. Bordons (2007). The h-index: advantages, limitations and its relation with other bibliometric indicators at the micro level. Journal of Informetrics 1(3), 193203, 2007.

V. Cothey (2007). Applying Egghe's general theory of the evolution of Information Production Processes to the World Wide Web. Proceedings of ISSI 2007. $11^{\text {th }}$ International Conference of the International Society for Scientometrics and Informetrics, CSIC, Madrid (Spain) (D. Torres-Salinas and H.F. Moed, eds.), 231240, 2007.

L. Egghe (2004). Positive reinforcement and 3-dimensional informetrics. Scientometrics 60(3), 497-509. Correction: Scientometrics 61(2), 283, 2004.

L. Egghe (2005). Power Laws in the Information Process: Lotkaian Informetrics. Elsevier, Oxford (UK), 2005.

L. Egghe (2006a). How to improve the h-index. Letter. The Scientist 20(3), 14, March 2006.

L. Egghe (2006b). Theory and practise of the g-index. Scientometrics, 69(1), 131-152, 2006. 
L. Egghe (2006c). An improvement of the h-index: the g-index. ISSI Newsletter 2(1), 8-9, 2006.

L. Egghe (2007a). General evolutionary theory of information production processes and applications to the evolution of networks. Journal of Informetrics 1(2), 115-122, 2007.

L. Egghe (2007b). Dynamic h-index: the Hirsch index in function of time. Journal of the American Society for Information Science and Technology 58(3), 452-454, 2007.

L. Egghe (2007c). Modelling successive h-indices. Scientometrics, to appear.

L. Egghe and I.K. Ravichandra Rao (2007a). The influence of the query formulation of a topic on its h-index: models and examples of the h-index of N-grams. Preprint.

L. Egghe and I.K. Ravichandra Rao (2007b). Study of different h-indices for groups of authors. Preprint.

L. Egghe and R. Rousseau (2006a). An informetric model for the Hirsch-index.

Scientometrics, 69(1), 121-129, 2006.

L. Egghe and R. Rousseau (2006b). Systems without low productive sources. Information Processing and Management 42(6), 1428-1441, 2006.

W. Glänzel (2006a). On the opportunities and limitations of the h-index. Science Focus 1(1), 10-11, 2006 (in Chinese).

W. Glänzel (2006b). On the h-index - a mathematical approach to a new measure of publication activity and citation impact. Scientometrics 67(2), 315-321, 2006.

W. Glänzel and O. Persson (2005). H-index for Price medallists. ISSI Newsletter 1(4), 15-18, 2005.

J.E. Hirsch (2005). An index to quantify an individual's scientific research output.

Proceedings of the National Academy of Sciences of the United States of America 102, 16569-16572, 2005.

J.E. Hirsch (2007). Does the h-index have predictive power ? arXiv:0708.0646v1 [physics.soc-ph] 6 Aug 2007.

C.W. Miller (2006). Superiority of the h-index over the impact factor for physics. arXiv:physics/0608183v1 17 Aug 2006.

C. Oppenheim (2007). Using the h-index to rank influential British researchers in information science and librarianship. Journal of the American Society for Information Science and Technology 58(2), 297-301, 2007.

G. Prathap (2006). Hirsch-type indices for ranking institutions' scientific research output. Current Science 91(11), 1439, 2006. 
I.K. Ravichandra Rao and R. Rousseau (2007). Empirical series of journal h-indices. Preprint.

R. Rousseau (1992). Concentration and diversity of availability and use in information systems: a positive reinforcement model. Journal of the American Society for Information Science 43(5), 391-395, 1992.

R. Rousseau (2007a). The influence of missing publications on the Hirsch index. Journal of Informetrics 1(1), 2-7, 2007.

R. Rousseau (2007b). A case study: evolution of JASIS' Hirsch index. Preprint.

G. Saad (2006). Exploring the h-index at the author and journal levels using bibliometric data of productive consumer scholars and business-related journals respectively. Scientometrics 69(1), 117-120, 2006.

M. Schreiber (2007). The influence of self-citation corrections on Egghe's g-index. arXiv:0707.4577v1[physics.soc-ph] 31 Jul 2007.

A. Schubert (2007). Successive h-indices. Scientometrics 70(1), 201-205, 2007.

A. Schubert and W. Glänzel (2007). A systematic analysis of Hirsch-type indices for journals. Journal of Informetrics 1(3), 179-184, 2007.

The STIMULATE6 Group (2007). The Hirsch index applied to topics of interest to developing countries. First Monday 12(2), 2007. http://www.firstmonday.org/issues/issues/issues12 2/stimulate/

R.S.J. Tol (2007). A rational, successive g-index applied to economics departments in Ireland. Preprint.

A.F.J. van Raan (2006). Comparison of the Hirsch-index with standard bibliometric indicators and with peer judgement for 147 chemistry research groups. Scientometrics 67(3), 491-502, 2006.

J.K. Vanclay (2007). On the robustness of the h-index. Journal of the American Society for Information Science and Technology 58(10), 1547-1550, 2007.

P. Vinkler (2007). Eminence of scientists in the light of the h-index and other scientometric indicators. Journal of Information Science 33(4), 481-491, 2007.

J. Wan, P. Hua and R. Rousseau (2007). The pure h-index: calculating an author's h-index by taking co-authors into account. Preprint. 